\title{
The Virtual Recreation of a Ming Dynasty Pailou Gate
}

\author{
Yi Ji \\ School of Art and Design \\ Guangdong University of Technology \\ China \\ jiyi001@hotmail.com
}

\author{
Sean Clark \\ Institute of Creative Technologies \\ De Montfort University \\ UK \\ seanc@cuttlefish.com
}

\section{INTRODUCTION}

In this paper and demonstration, we present a Virtual Reality reconstruction of a 500-year-old 'pailou' gate, or memorial archway, that is currently located at the Guangdong University of Technology (GDUT), China. The small roofed structure was built during the Ming Dynasty in the Shanxi Province. In 2013 it was acquired by GDUT and in 2014 it was installed in the courtyard of one of the main University buildings on the Dongfeng Road Campus in Guangzhou.

Based in the School of Art and Design, our project had two goals:

1. To accurately model the archway for general presentation and on-going study.

2. To create an interactive Virtual Reality experience that would locate the building in a reimagining of its original historical context.

\section{THE PAILOU GATE AT GDUT}

The gate is currently located in the courtyard of the library building on the GDUT Dongfeng Road campus. It is presented in a small garden area with two ornamental lions and an information board (Figure 1).

The building is part of a historic Chinese architectural tradition that is similar to the western tradition of a memorial arch. The gate has both symbolic and commemoration functions that are regarded as important elements of Chinese cultural heritage in both the urban and rural landscape.

This particular pailou gate was built in the Ming Dynasty in Shanxi province and was located there for around 500 years. Although some elements

were replaced in the early Qing Dynasty, it has retained the integrity of the original Ming Dynasty structure.

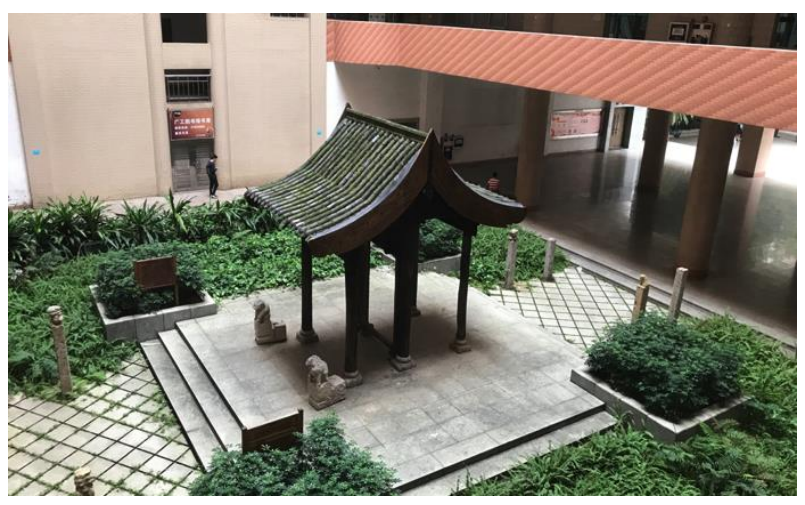

Figure 1: The Pailou Gate at the Guangdong University of Technology.

In addition to its functional and decorative role, the gate also expresses the prevailing ideas of the owners. The calligraphy of the plaque "She De Wu Zi" indicates the owner's principle of doing things diligently and pragmatically, as well as the their charitable intents.

\section{THE 3D MODEL}

The process of modelling the gate in 3D began with the manual process of photographing and measuring all parts of the structure. Reference was also made to formal Chinese architectural texts describing the construction of buildings for the era in order to understand the internal structure of the wooden joints.

The model was then created in 3D Studio Max and eventually consisted of approximately 100,000 polygons (Figure 2). Simple textures were added and the resultant model was then readied for inclusion in the Virtual Realty experience. 


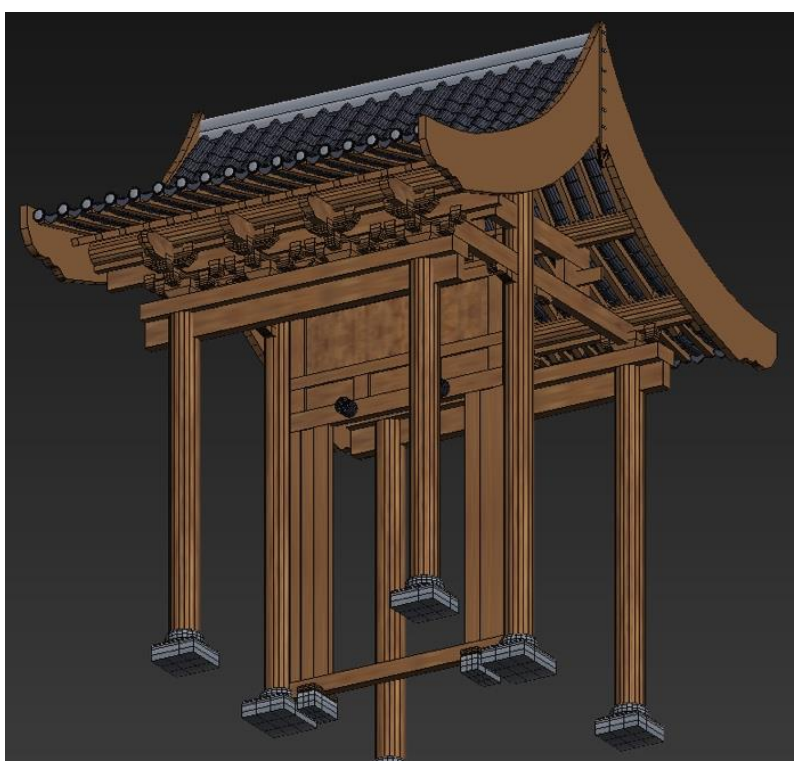

Figure 2: The 3D model of the pailou gate.

\section{THE VIRTUAL REALITY ENVIRONMENT}

Having achieved the first goal of creating a 3D model of the gateway, we undertook research in to the original locations of such archways in the Ming Dynasty and the types of objects that would have been found nearby at the time. This research was used to inform the creation of a Virtual Reality environment built using the Unreal games engine (Figure 3).

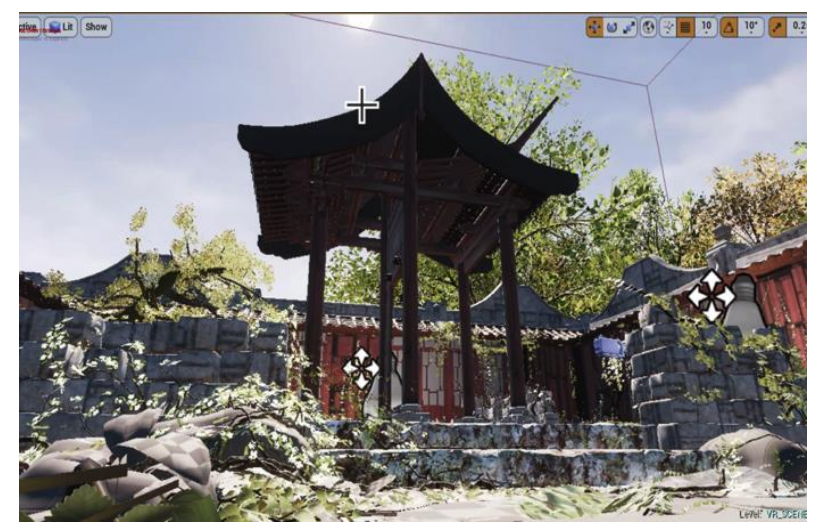

Figure 3: The 3D placed in a Virtual Reality environment.

Central to this environment was the creation of a courtyard that would have been typical of the original setting (Figure 4).

The archway and the environment, together with information about the cultural and architectural significance of the structure were then packaged and presented as a VR application for HTC Vive and Google Cardboard.

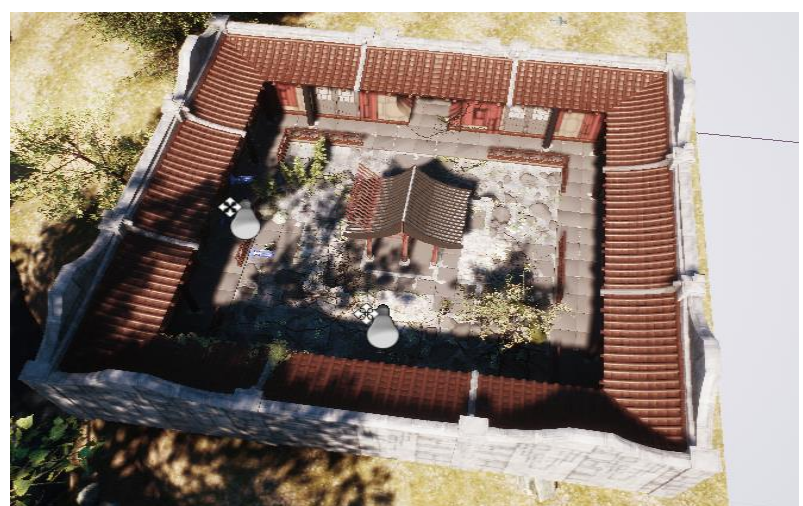

Figure 4: For the virtual environment the gate was placed within a historic building courtyard.

\section{INSIGHTS}

The process of modelling and contextualising the pailou gate was revealing as to the nature of the original architecture and has given the team insights in to the construction of such buildings. We intend to share these insights further with architect colleagues and will report on it further.

Our desire to keep this as a live project, with regular updates, has revealed limitations in the technology used. While the use of the Unreal games engine has enabled us to create a realistic environment in which to locate the gate, it does require us to release a full app update with every set of enhancements made.

We have therefore been investigating alternative distribution methods for the VR environment. The most promising of these is to use the WebVR platform (2017). This allows the project to be shared as a WebVR web page and updated at will

Both the Unreal and WebVR versions of the project will be demonstrated at the EVA London 2018 conference. The latest versions of the applications can be downloaded from the project web page (Clark 2018).

Thanks to Li Wanli, Zheng Ziyue, Zheng Kaiyang and Kuang Shuqin from GDUT for their work on the project and the School of Art and Design at GDUT for their on-going support.

\section{REFERENCES}

Clark, S. (2018) The Pailou Gate Project. http://interactdigitalarts.uk/the-pailou-gate (retrieved 18 March 2018).

WebVR (2017). Editor's Draft: 12 December 2017. https://immersive-web.github.io/webvr/ (retrieved 18 March 2018). 